This is a pre-publication version of the following article:

Barry, J. A., Qu, F., \& Hardiman, P. J. (2018). An exploration of the hypothesis that testosterone is implicated in the psychological functioning of women with polycystic ovary syndrome (PCOS). Medical Hypotheses, 110, 42-45.

\title{
An exploration of the hypothesis that testosterone is implicated in the psychological functioning of women with polycystic ovary syndrome (PCOS).
}

Running title: Linking testosterone \& psychology in PCOS

\author{
JA Barry, $\mathrm{PhD},{ }^{1 *} \mathrm{FQu}, \mathrm{PhD},{ }^{2}$ and $\mathrm{PJ}$ Hardiman, $\mathrm{MD} .^{3}$ \\ ${ }^{1}$ Clinical, Educational and Health Psychology, University College London, 1-19 Torrington \\ Place, London WC1E 6BT, UK. \\ ${ }^{2}$ Women's Hospital, School of Medicine, Zhejiang University, Hangzhou, 310006, China \\ ${ }^{3}$ Institute for Women's Health, University College London Medical School, London NW3 \\ $2 \mathrm{PF}$
}

Corresponding authors: JA Barry, Clinical, Educational and Health Psychology, University College London, 1-19 Torrington Place, London WC1E 6BT, UK. Tel: 02077940500 ext. 34081; Fax: 0207830 2261. E-mail: john.barry@ucl.ac.uk

\section{$\underline{\text { OR }}$}

Dr Fan QU, Associate Professor, Women's Hospital, School of Medicine, Zhejiang University, No.1 Xueshi Road, Hangzhou, Zhejiang, 310006, China. Tel: +86-571-87061501; Fax: +86-571-87061878 ; Mobile: +86 13429119143; E-mail: qufan43@outlook.com

No specific funding was allocated for this paper 


\begin{abstract}
One of the diagnostic features of polycystic ovary syndrome (PCOS) is elevation of the androgen, testosterone. It is known that women with PCOS are more likely to suffer from psychological problems, especially anxiety and depression, than other women. However, little is known of how much of this is due to testosterone, and if so, what the mechanism(s) might be.
\end{abstract}

This study explores the hypothesis that testosterone impacts women with PCOS both directly and indirectly, via testosterone currently in the bloodstream and through prenatal exposure. It is hypothesized that direct effects occur when testosterone acts directly upon receptors; indirect effects occur where the impact of testosterone is mediated via another variable; activational effects are ephemeral and are caused by testosterone in the bloodstream; organizational effects occur prenatally and cause permanent changes.

Four pathways are hypothesized in this paper: 1/ a direct and activational pathway which improves mental rotation ability; 2 / an indirect and activational pathway, whereby distress is caused when the physiological symptoms of testosterone are experienced; $3 /$ an indirect and organizational effect on mood, where elevated prenatal testosterone predisposes women with PCOS to low blood sugar levels and thus low mood; 4/ and finally, it is suggested that the pathway from biology to psychology can be travelled in reverse, with an direct activational effect of relaxation training on the reduction of adrenal androgens.

Testing these hypotheses has important implications for our understanding of PCOS, and our ability to treat this condition more effectively.

Keywords: polycystic ovary syndrome (PCOS); testosterone; psychology; depression; activational effect; organizational effect. 


\section{INTRODUCTION}

Roughly $8 \%$ of women have polycystic ovary syndrome (PCOS) [1]. Elevation of testosterone is one of the diagnostic criteria of PCOS. The typical symptoms of PCOS are subfertility, acne, hirsutism, obesity, and insulin resistance. Women with PCOS are more at risk of cancer [2], and coronary heart disease and stroke [3] than are other women.

In recent years it has become recognized that women with PCOS may be more susceptible than other women to a range of psychological issues [4], especially anxiety and depression [5]. However according to some of the leading researchers in PCOS: "...there is still a long way until we establish definitive causative links between the physiological and the psychological phenomena that take place in PCOS" [6, p.92].

The aim of this paper is outline testable, evidence-based hypotheses in regards psychological aspects of PCOS.

\section{HYPOTHESES}

In medicine, PCOS has traditionally been regarded as a fertility issue [7]. Today PCOS is seen a little more holistically, and although the psychological aspects of PCOS have begun to be recognised in recent years, this awareness is largely limited to anxiety and depression, which are often explained as being the understandable reaction to having a condition with distressing symptoms such as obesity. It has been suggested [6] that we have barely scratched the surface of the biological underpinnings of the psychological issues related to PCOS. The hypotheses presented in this paper offer a significant step forward, in not only recognising issues beyond anxiety and depression, but also in seeing psychological issues in PCOS from a psychobiological perspective rather than a medical perspective.

Based on animal models, and on evidence from congenital adrenal hyperplasia in humans [8], there are several ways in which testosterone might have an effect on women with PCOS. In this literature, the timing of exposure to testosterone is recognized as important, and is 
usually discussed in terms of activational effects, which result from circulating levels of hormones in the postnatal bloodstream and are ephemeral, and organizational effects, which occur at critical or sensitive periods of prenatal or early neonatal life and are permanent. Pathways might be direct, where testosterone acts directly upon receptors, or indirect, where the impact of testosterone is mediated by another variable. Effects are sometimes combined, for example, and effect might be both direct and activational. The various possible mechanisms and pathways have been until now little explored in relation to psychological aspects of PCOS.

It is important to be able to understand the psychobiological parameters of PCOS because it might help us to devise more effective treatments for psychological and physiological problems in PCOS. This in turn might potentially reduce the economic burden of PCOS, estimated to cost at least $\$ 13.9$ billion annually in the United States [9].

We hypothesise that:

1/ Increased mental rotation ability in PCOS is caused by a direct and activational effect of testosterone

2/ Distress caused by living with the unwelcome symptoms of PCOS is an indirect activational effect of testosterone

3/ Mood issues in PCOS are additionally likely to be partly an organizational effect of elevated prenatal testosterone on the metabolic system, causing a tendency to low blood sugar and thus low mood.

4/ Relaxation training will reduce the production of adrenal androgens via a direct activational effect. 


\section{EVALUATION OF THE HYPOTHESIS}

\section{Hypothesis 1: Increased mental rotation ability in PCOS is caused by a direct and activational effect of testosterone}

This pathway is hypothesised to explain the findings of a study [10] in which 69 women with PCOS and 41 subfertile controls completed a standard three-dimensional mental rotation task [11]. Previous research has found that performance on this task shows a sex difference [12], and is related positively with testosterone [13]. Serum testosterone concentrations were measured using the Roche Elecsys system 1010/2010 Modular E170, a direct electrochemiluminescence (ECLIA) methodology (Roche Diagnostics, Indianapolis). Women with PCOS scored higher than controls on the mental rotation task, with a median (range) of $3.00(0-9)$ and $2.00(0-8)$, respectively (Mann-Whitney $U=1147.500, \mathrm{~N}_{1}=69$, $\left.\mathrm{N}_{2}=41, \mathrm{p}<.047\right)$. In the PCOS group but not controls, testosterone was positively correlated with mental rotation scores $\left(r_{s}=.376, \mathrm{~N}=56 p<.002\right)$ and estradiol was negatively correlated with mental rotation scores $\left(r_{s}=-.473, \mathrm{~N}=29, p<.010\right)$. Other factors, including general intelligence and social class, did not account for these effects, leaving the direct activational effect of testosterone in women with PCOS as a plausible explanation.

It is also possible that this direct / activational effect has its etiology in prenatal exposure to testosterone in PCOS [14], masculinising brain morphology (see Fig 1, and Supplementary Fig 1a). According to this hypothesis, prenatal exposure would increase the expression of androgen receptors in cortical areas, the hippocampus and parietal lobe, which are associated with visuospatial ability. This greater abundance of receptors would facilitate the uptake of circuiting testosterone, increasing mental rotation task performance. However the effect of PCOS on mental rotation ability [10] was seen only in those women with elevated testosterone levels; women with PCOS and normal testosterone levels did not show 
enhanced mental rotation ability. However the evidence in this study is largely correlational, and the hypothesis could be better tested by comparing the mental rotation ability of women with PCOS before and after taking androgen-lowering medication, such as spironolacatone, which would be hypothesised to reduce mental rotation ability.

The alternative hypothesis, that mental rotation differences are due to organisational effects of prenatal testosterone exposure on brain structure, could also be tested by mapping androgen receptors using positron emission tomography (PET) in women with PCOS. Androgen receptors could be mapped both in terms of distribution [15] and degree of activity [16] to find out whether there are differences between women with PCOS compared to other women, and whether such differences are related to scores on mental rotation tasks.

\section{2/ Distress is caused by living with the unwelcome symptoms of PCOS, an indirect activational effect of testosterone}

This pathway is hypothesised to explain the findings of a study [17] in which 76 women with PCOS and 49 subfertile controls completed the Hospital Anxiety and Depression Scale (HADS) [18], PCOS Quality of Life Questionnaire (PCOSQ) [19], Eysenck Personality Questionnaire (EPQ) [20] and the Aggression Inventory [21]. Assays were performed as described in [10]. Controlling for age and body mass index (BMI) using multivariate analysis of covariance (MANCOVA), it was found that women with PCOS were significantly more neurotic (had difficulty coping with stress) than controls $(\mathrm{F}=3.95, \mathrm{p}<.05)$, and had more quality of life problems related to acne $(\mathrm{Z}=-2.75, \mathrm{p}<.01)$. Women with PCOS also had more anger symptoms, and a greater tendency to withhold anger. Testosterone was not generally correlated with mood states, and in regression of testosterone on neuroticism in PCOS, $R$ Square change analysis of the data (not previously published) shows that testosterone explained only $4 \%$ of neuroticism scoring. In contrast, the QoL impact of hirsutism explained 
$16 \%$ of scoring in neuroticism, QoL for acne explained 13\% of scoring in neuroticism, and QoL for menstrual problems explained 10\% of scoring in neuroticism; these three QoL subscales combined explained $25 \%$ of scoring in neuroticism. The pattern of relationships was similar when anxiety and depression, rather than neuroticism, were the dependent variables. Thus mood disturbance in PCOS is likely to be partly due to an indirect activational effect of testosterone, in that testosterone causes distressing physical symptoms (skin problems, subfertility etc) which in turn have a negative impact mood (see Fig 1, and Supplementary Fig 1b). A similar interpretation can be made regarding the impact of obesity on depression in PCOS; evidence for this is the meta-analysis finding [5] that the higher levels of anxiety and depression in women with PCOS compared to other women were reduced by a small amount (by a Hedge's $g$ of 0.15 and 0.12 , respectively) when body mass index (BMI) was taken into account.

Hypothesis 2 could be tested experimentally by comparing the PCOS symptom QoL scores of women with PCOS before and after being randomly allocated to either androgenreducing medication (such as spironolacatone) or placebo, to test whether QoL was more strongly correlated with objective symptom measures (e.g. BMI, or the Ferriman-Gallway measure of hirsutism) than with circulating testosterone level. This test would be enhanced by having double blind crossover design.

\section{3/ Mood issues in PCOS are partly an organizational effect of elevated prenatal testosterone on the metabolic system, causing a tendency to low blood sugar and thus low mood.}

This pathway is hypothesised to explain the findings of an online survey of 24 women with PCOS, 299 healthy control women, 47 women who possibly had undiagnosed PCOS [22]. The groups were compared for symptoms of mood and behavioral symptoms (e.g. tiredness, 
anxiety, low mood) typical of reactive hypoglycemia (i.e. low blood glucose experiences after meals), using the Hypoglycemia Symptom Checklist -7 (HSC-7) [22]. Controlling for age and body mass index (BMI) using between-groups analysis of covariance (ANCOVA), the women with PCOS scored significantly higher than the other groups on all of the mood outcomes measured (minimum $p<0.001$ ). This study shows that mood issues in PCOS are likely to, in part, result from hypoglycaemia, which in turn is probably the result of an organizational effect of elevated prenatal testosterone on the metabolic system, causing insulin resistance and glucose imbalance in adults with PCOS (see Fig 1, and Supplementary Fig 1b).

Hypothesis 3 could be tested experimentally by altering blood glucose levels in women with PCOS and seeing how much this impacts mood. To improve the ecological validity of the study, a nutritional intervention such as a low GI diet [23] could be used to reduce any tendency to reactive hypoglycaemia. Also, rather than a self-reported measure of psychological symptoms of hypoglycaemia, a continuous blood glucose monitor should be used to improve data collection and validity.

\section{4/ Relaxation training will reduce testosterone levels, thus improving mood}

Psychological distress can activate the adrenal glands, which in turn produce biochemicals such as cortisol and andrenal androgens (such as DHEAS and androstendione) [24]. Furthermore, women with PCOS may be more sensitive to the stress hormone adrenocorticotropic hormone (ACTH), leading to an abnormally high production of adrenal androgens $[25,26,27]$. Thus a vicious cycle may occur, where adrenal androgens contribute to the distressing symptoms of PCOS, and distress in reaction to the symptoms might lead to an increase in adrenal androgens.

Psychological relaxation techniques can non-pharmacologically reduce hypothalamicpituitary axis (HPA) activity, and a recent study of mindfulness training for women with 
PCOS found a significant reduction in cortisol, stress and depression, though no improvement in quality of life (QoL) [28].

This psychobiological pathway is hypothesised to explain the findings of pilot the study by [29], in which the participants were 13 women with PCOS, aged between 19 and 33. Relaxation treatment consisted of six weekly sessions and a follow-up session three months later. Questionnaires (HADS and PCOSQ) and blood samples were taken at roughly the same time, and for each participant, at roughly the same time of day (early evening).

Questionnaires were completed before the first and sixth session, and at follow-up; blood samples were completed before and after the first and sixth session, and before and after follow-up. Androgens were assayed using tandem mass spectrometry. There was a significant reduction in DHEAS from before to after the intervention at week $1(t=1.921$, d.f. $=9, P<.044)$ and before to after week $6(t=4.301$, d.f. $=10, P<.001)$. There was also a significant reduction in androstenedione from before to after week $6(t=2.766$, d.f. $=10, P<.010)$. There was no change in free or total testosterone. There was a significant reduction in anxiety from week 1 to week $6(t=$ 2.01 , d.f. $=10, P<.037)$. There was a significant reduction in depression from week 1 to week $6(t=$ 2.10, d.f. $=10, P<.034)$ and from week 1 to follow-up at 3 months $(t=2.34$, d.f. $=8, P<.024)$. There was a non-significant improvement in QoL from week 1 to week 6. This study indicates that relaxation can reduce adrenal androgen levels, demonstrating that causality works not only from androgen to mood (as seen in Hypothesis 2, above), but from mood to androgen via a direct activational effect (see Fig 1, and Supplementary Fig 1c). (An alternative hypothesis is that the relaxation training itself improved mood; this is possible but ignores the changes in hormones).

Hypothesis 4 could be tested experimentally by using a randomized controlled trial design and some other improvements (e.g. a longer, and thus potentially more powerful, relaxation session, having more frequent sessions, a control group, and a larger sample size). 


\section{CONSEQUENCES OF THE HYPOTHESES AND DISCUSSION}

Other hypotheses regarding PCOS should be considered [e.g. 30], but if the hypotheses presented in the present paper are supported, it would have specific implications for how we approach the treatment of PCOS. Exploration of this kind could yield clinical benefits for women with PCOS, for example, relaxation methods might be prescribed to increase control of adrenal androgens in PCOS, especially patients who experience troubling side effects of pharmacological alternatives. Or more radical interventions might be considered, for example, a proactive / preventative strategy might be taken to reduce the prenatal development of PCOS (e.g. administration of an anti-androgen during pregnancy with a female fetus) and a reactive strategy, where the reduction of testosterone levels would be one of the key aims of therapy in women. Obviously a rigorous risk-assessment of the pregnancy intervention would be necessary.

Despite the fact that so many women are affected by PCOS, the NIH has failed to grasp the issue of the psychological needs of these women [31]. Thus it is little wonder that women with PCOS suffer from long-term mental health issues [32]. As stated by Papalou \& Diamanti-Kandarakis in regard to mental health in PCOS: "I hope that with a coordinated, international effort, we would make a step further in comprehending eventually the unique pathophysiological pathway of PCOS" [6, p.92].

\section{Acknowledgements}

One study [29] was partly supported by a grant from the Royal Free Hospital Charity.

\section{Declaration of conflicts of interest}

The authors have no conflicts of interests to declare 


\section{References}

[1] Azziz R,Woods KS, Reyna R, Key TJ, Knochenhauer ES, Yildiz BO. The prevalence and features of the polycystic ovary syndrome in an unselected population. J Clin Endocrinol Metab 2004;89:2745-2749.

[2] Barry JA, Azizia MM, Hardiman PJ. Risk of endometrial, ovarian and breast cancer in women with polycystic ovary syndrome: a systematic review and meta-analysis. Hum Rep Update 2014;1:748-58.

[3] Anderson SA, Barry JA, Hardiman PJ. Risk of coronary heart disease and risk of stroke in women with polycystic ovary syndrome: A systematic review and metaanalysis. Int J Cardiol 2014;176:486-7

[4] Himelein MJ, Thatcher SS. Polycystic ovary syndrome and mental health: a review. Obs \& Gyn Surv 2006;1:723-32.

[5] Barry JA, Hardiman PJ, Saxby BK, Kuczmierczyk A. Testosterone and mood dysfunction in women with polycystic ovarian syndrome compared to subfertile controls. J Psychosom Obstet Gynecol 2011; 32:104-111.

[6] Papalou O, \& Diamanti-Kandarakis E. The role of stress in PCOS. Expert Rev Endocrinol Metab 2017;12:87-95.

[7] Hunter MH, \& Sterrett JJ. Polycystic ovary syndrome: it's not just infertility. Am Fam Physician 2000;62:1079-88.

[8] Hines M. Brain Gender. Cambridge: Cambridge University Press. 2004

[9] Azziz R. Polycystic ovary syndrome is a family affair. J Clin Endocrinol Metab 2008;93:1579.

[10] Barry JA, Parekh HSK, Hardiman PJ. Visual-spatial cognition in women with polycystic ovarian syndrome: the role of androgens. Hum Reprod 2013;28:2832-2837. 
[11] Peters M, Laeng B, Latham K, Jackson M, Zaiyouna R, Richardson C. A redrawn Vandenberg and Kuse mental rotations test-different versions and factors that affect performance. Brain Cogn 1995; 28:39-58.

[12] Voyer D. Time limits and gender differences on paper-and-pencil tests of mental rotation: a meta-analysis. Psychonom Bull Rev 2011;18: 267-277

[13] Aleman A, Bronk E, Kessels RP, Koppeschaar HP, \& van Honk J. A single administration of testosterone improves visuospatial ability in young women. Psychoneuroendocrinology 2004;29:612-617.

[14] Barry JA, Kay AR, Navaratnarajah R, Iqbal S, Bamfo JE, David AL, Hines M, Hardiman PJ. Umbilical vein testosterone in female infants born to mothers with polycystic ovary syndrome is elevated to male levels. J Obstet Gynaecol 2010;1: 444446.

[15] Garg S, Doke A, Black KW, Garg PK. In vivo biodistribution of an androgen receptor avid PET imaging agent 7-alpha-fluoro-17 alpha-methyl-5-alpha-dihydrotestosterone ([(18)F]FMDHT) in rats pretreated with cetrorelix, a GnRH antagonist. Eur J Nucl Med Mol Imaging 2008:35:379-385

[16] Evans M J, Smith-Jones PM, Wongvipat J, Navarro V, Kim S, Bander NH, Sawyers CL. Noninvasive measurement of androgen receptor signaling with a positron-emitting radiopharmaceutical that targets prostate-specific membrane antigen. Proc Natl Acad Sci 2011:108:9578-9582.

[17] Barry JA, Hardiman PJ, Saxby BK, Kuczmierczyk A. Testosterone and mood dysfunction in women with polycystic ovarian syndrome compared to subfertile controls. J Psychosom Obstet Gynecol;32:104-111.

[18] Snaith RP, Zigmond AS. The hospital anxiety and depression scale. Br Med J Clin Res Ed 1986;292: 344. 
[19] Cronin L, Guyatt G, Griffith L, Wong E, Azziz R, Futterweit W, Cook D, Dunaif A. Development of a health-related quality-of-life questionnaire (PCOSQ) for women with polycystic ovary syndrome (PCOS) J Clin Endocrinol Metab 1998;83:1976-1987.

[20] Eysenck HJ, Eysenck SBG. EPQ-R short scale. London \& Hodder Stoughton Educ. 1991

[21] Buss AH, Perry M. The aggression questionnaire. J Pers Soc Psychol 1992; 63:452.

[22] Barry JA, Bouloux P, Hardiman PJ. The impact of eating behavior on psychological symptoms typical of reactive hypoglycemia. A pilot study comparing women with polycystic ovary syndrome to controls. Appetite. 2011;57:73-6.

[23] Marsh KA, Steinbeck KS, Atkinson FS, Petocz P, Brand-Miller JC. Effect of a low glycemic index compared with a conventional healthy diet on polycystic ovary syndrome. Am J Clin Nutrition 2010;92:83-92.

[24] Leowattana W. DHEAS as a new diagnostic tool. Clin Chim Acta 2004;341:1-15.

[25] McKenna TJ, Cunningham SK. Adrenal androgen production in polycystic ovary syndrome. Eur J Endocrinol Fed Endocr Soc 1995;133:383-9.

[26] Milutinović DV, Macut D, Božić I, Nestorov J, Damjanović S, Matić G. Hypothalamic-pituitary-adrenocortical axis hypersensitivity and glucocorticoid receptor expression and function in women with polycystic ovary syndrome. Exp Clin Endocrinol Diabetes Off J Ger Soc Endocrinol Ger Diabetes Assoc 2011;119:636-43.

[27] Moran C, Reyna R, Boots LS, Azziz R. Adrenocortical hyperresponsiveness to corticotropin in polycystic ovary syndrome patients with adrenal androgen excess. Fertil Steril 2004;81:126-31.

[28] Stefanaki C, Bacopoulou F, Livadas S, Kandaraki A, Karachalios A, Chrousos GP, et al. Impact of a Mindfulness Stress Management Program on Stress, Anxiety, Depression and Quality of Life in Women with Polycystic Ovary Syndrome: a 
Randomized Controlled Trial. Stress. 2014;0:1-34

[29] Barry JA, Leite N, Sivarajah N, Keevil B. Owen L, Miranda LCS, Qu F, and Hardiman PJ. Psychological relaxation significantly improves anxiety, depression, quality of life and lowers androgen levels in women with polycystic ovary syndrome (PCOS): a pilot study. Contemp Hypn \& Integr Ther 2017; 31:14-31

[30] Wawrzkiewicz-Jałowiecka A, Kowalczyk K, Pluta D, Blukacz Ł, Madej P. The role of aquaporins in polycystic ovary syndrome-A way towards a novel drug target in PCOS. Med Hypotheses 2017;102:23-7.

[31] Sanchez N. Suitability of the National Health Care Surveys to Examine Behavioral Health Services Associated with Polycystic Ovary Syndrome. J Behav Health Serv Res 2016;13:1-7.

[32] Karjula S, Morin-Papunen L, Auvinen J, Ruokonen A, Puukka K, Franks S, Järvelin MR, Tapanainen JS, Jokelainen J, Miettunen J, Piltonen TT. Psychological Distress Is More Prevalent in Fertile Age and Premenopausal Women with PCOS Symptoms-15yr Follow-up. J Clin Endocrinol Metab 2017;92:83-92 
Figure 1. A model of the suggested mechanisms underlying psychological problems in PCOS. The double headed shaded arrows indicate bidirectional causation. An interactive version of this diagram is available at Supplementary Fig. 1a-c [http://www.medical-hypotheses.com/action/showFullTextImages?pii=S03069877\%2817\%2930571-6] which highlights each pathway.

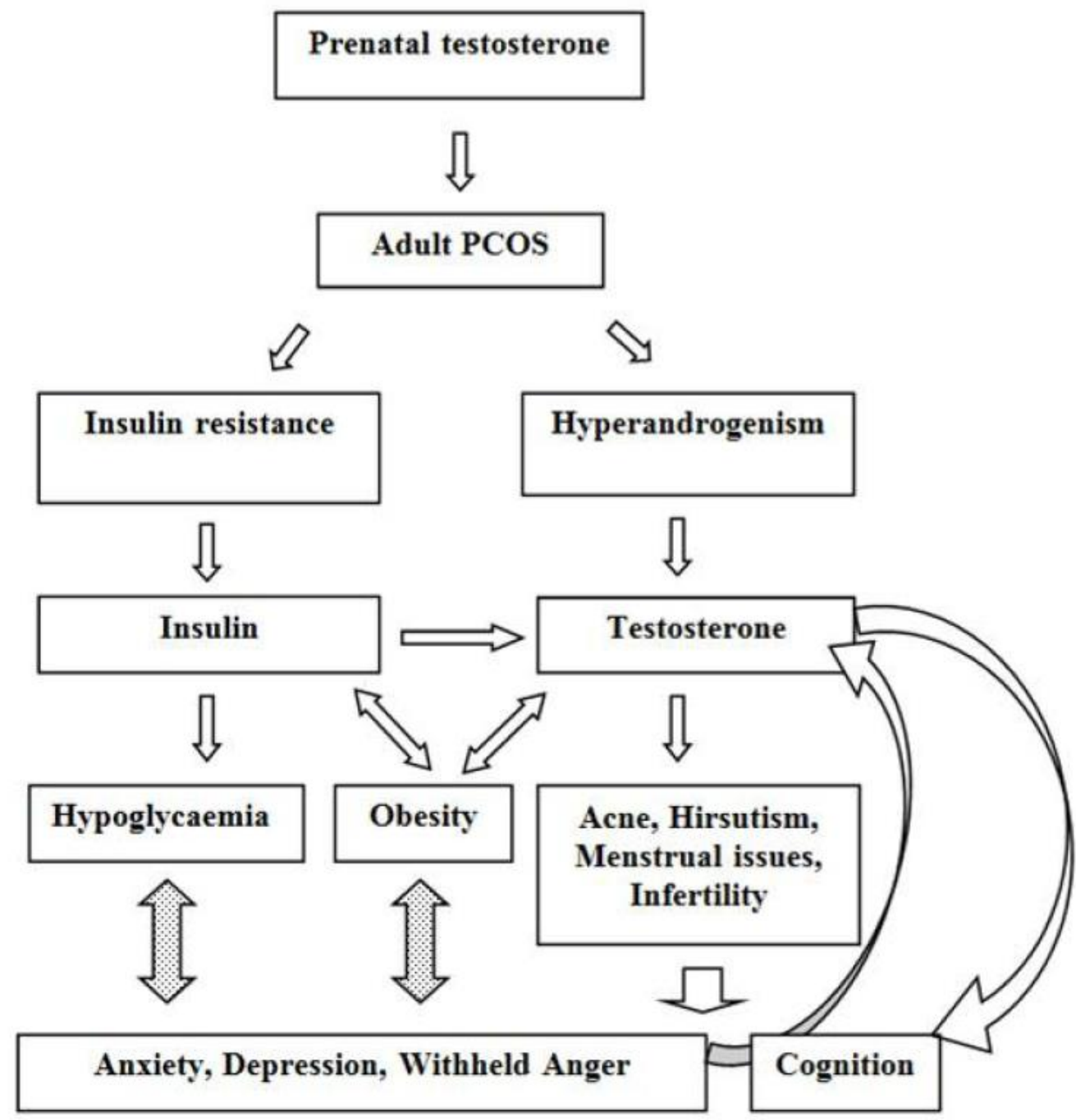

